\title{
Disjoint Edges in Geometric Graphs
}

\author{
N. Alon ${ }^{1 *}$ and P. Erdös ${ }^{2}$ \\ ${ }^{1}$ Department of Mathematics, Sackler Faculty of Exact Sciences, \\ Tel Aviv University, Ramat Aviv, Tel Aviv, Israel \\ ${ }^{2}$ Mathematical Institute of the Hungarian Academy of Sciences, \\ Budapest, Hungary
}

\begin{abstract}
Answering an old question in combinatorial geometry, we show that any configuration consisting of a set $V$ of $n$ points in general position in the plane and a set of $6 n-5$ closed straight line segments whose endpoints lie in $V$, contains three pairwise disjoint line segments.
\end{abstract}

A geometric graph is a pair $G=(V, E)$, where $V$ is a set of points (=vertices) in general position in the plane, i.e., no three on a line, and $E$ is a set of distinct, closed, straight line segments, called edges, whose endpoints lie in $V$. An old theorem of the second author [Er] (see also [Ku] for another proof), states that any geometric graph with $n$ points and $n+1$ edges contains two disjoint edges, and this is best possible for every $n \geq 3$. For $k \geq 2$, let $f(k n)$ denote the maximum number of edges of a geometric graph on $n$ vertices that contains no $k$ pairwise disjoint edges. Thus, the result stated above is simply the fact $f(2, n)=n$ for all $n \geq 3$. Kupitz [Ku] and Perles [Pe] (see also [AA]) raised the problem of determining or estimating $f(k n)$ for $k \geq 3$. In particular, they asked if $f(3, n) \leq$ $O(n)$. This specific problem, of determining or estimating $f(3, n)$, was already mentioned in 1966 by Avital and Hanani [AH], and it seems it was a known problem even before that. In this note we answer this question by proving the following.

Theorem 1. For every $n \geq 1, f(3, n)<6 n-5$, i.e, any geometric graph with $n$ vertices and $6 n-5$ edges contains three pairwise disjoint edges.

\footnotetext{
* Research supported in part by an Allon Fellowship and by a Bat Sheva de-Rothschild grant.
} 
Before proving this theorem we note that clearly

$$
f(3, n)=\left(\begin{array}{l}
n \\
2
\end{array}\right) \text { for } n \leq 5
$$

and the best-known lower bound for $n \geq 6$, given by Perles [Pe], is

$$
f(3, n) \geq \begin{cases}\frac{5}{2} n-\frac{5}{2} & \text { for odd } n \geq 5 \\ \frac{5}{2} n-4 & \text { for even } n \geq 2\end{cases}
$$

To prove inequality (1) for odd $n$ consider the geometric graph $G_{n}$ whose $n$ vertices are the $n-1$ points $v_{3}=(\cos (2 \pi j /(n-1)), \sin (2 \pi j /(n-1))), 0 \leq j<n-1$, together with the additional point $u=(\varepsilon, \delta)$ where $\varepsilon$ and $\delta$ are small numbers chosen so that $\left\{v_{0}, \ldots, v_{n-2}, u\right\}$ is in general position. The edges of $G_{n}$ are the $\frac{5}{2}(n-1)$ line segments

$$
\begin{aligned}
\left\{\left[u, v_{j}\right]: 0 \leq j<n-1\right\} & \\
\cup & \left\{\left[v_{j}, v_{j+(n-3) / 2}\right],\left[v_{j}, v_{j+(n-1) / 2}\right],\left[v_{j}, v_{j+(n+1) / 2}\right]: 0 \leq j<n-1\right\},
\end{aligned}
$$

where all indices are reduced modulo $n-1$. We can easily check that if $\varepsilon$ and $\delta$ are sufficiently small then $G_{n}$ contains no three pairwise disjoint edges. Thus $f(3, n) \geq \frac{5}{2} n-\frac{5}{2}$ for every odd $n \geq 5$. For even $n$, let $G_{n}$ be the geometric graph obtained from $G_{n+1}$ by deleting one of its vertices of degree 4 . Then $G_{n}$ has $\frac{5}{2} n-4$ edges and contains no three pairwise disjoint edges. This establishes (1). On the other hand, Perles [Pe] showed that every geometric graph whose $n$ vertices are the vertices of a convex $n$-gon in the plane, with more than $(k-1) n$ edges, contains $k$ pairwise disjoint edges. In particular, in the convex case $2 n+1$ edges guarantee three pairwise disjoint edges. Comparing this with (1) we conclude that the convex case differs from the general one.

Our final remark before the proof of Theorem 1 is that a special case of one of the results in [AA] implies that, for every $k=o(\log n), f(k, n)=o\left(n^{2}\right)$. It is very likely that, for every fixed $k, f(k, n)=O(n)$, and that, for every $k=o(n)$, $f(k, n)=o\left(n^{2}\right)$, but this remains open.

Proof of Theorem 1. Let $G$ be a geometric graph with $n$ vertices and $6 n-5$ edges. We must show that $G$ contains three pairwise disjoint edges. It is first convenient to apply an affine transformation on the plane, in order to make all the edges of $G$ almost parallel to the $x$-axis. This is done by first choosing the $x$-axis so that any two distinct points of $G$ have different $x$-coordinates, and then, by rescaling the $y$-coordinates so that the difference between the $x$-coordinates of any two distinct points of $G$ is at least 1000 times bigger than the difference between their $y$-coordinates. Since any affine transformation maps disjoint segments into disjoint segments we may apply the above transformations, and hence may assume that $G$ satisfies the following:

The small angle between any edge of $G$ and the $x$-axis is less than $\pi / 200$. 
We now define the clockwise derivative and the counterclockwise derivative of an arbitrary geometric graph. Let $H=(V, E)$ be a geometric graph and let $e=[u, v]$ be an edge of $H$. We say that $e$ is clockwise good at $u$ if there is another edge $e^{\prime}=\left[u, v^{\prime}\right]$ of $H$ such that the directed line $\overrightarrow{u v^{\prime}}$ is obtained from $\overrightarrow{u v}$ by rotating it clockwise around $u$ by an angle smaller than $\pi / 100$. If $e$ is not clockwise good at $u$, we say that it is clockwise bad at $u$. The edge $e=[u, v]$ is clockwise good if it is clockwise good at both $u$ and $v$. The clockwise derivative of $H$, denoted by $a H$, is the geometric graph whose set of vertices is the set of all vertices of $H$, and whose set of edges consists of all clockwise good edges of $H$. The notions of an edge $e=[u, v]$ which is counterclockwise good at $u$ and that of an edge which is counterclockwise good are defined analogously. The counterclockwise derivative of $H$, denoted by $H \partial$, is also defined in an analogous manner.

Claim 1. Let $G=(V, E)$ be a geometric graph with $n \geq 2$ vertices and $m$ edges satisfying (2). Then the number of edges of $\partial G$ is at least $m-(2 n-2)$. Similarly, the number of edges of $G \partial$ is at least $m-(2 n-2)$.

Proof. We prove the assertion for $\partial G$. The proof for $G \partial$ is analogous. Let $v \in V$ be an arbitrary vertex of $G$. We claim that the number of edges of the form $[v, u]$ of $G$ which are clockwise bad at $v$ does not exceed 2 . Indeed, assume this is false and let $\left[v, u_{1}\right],\left[v, u_{2}\right],\left[v, u_{3}\right]$ be three such edges. Without loss of generality, assume that the $x$-coordinates of $u_{1}$ and $u_{2}$ lie in the same side of the $x$-coordinate of $v$. By (2), the angle between $\left[v, u_{1}\right]$ and $\left[v, u_{2}\right]$ is smaller than $\pi / 100$, and hence at least one of these two edges is clockwise good at $v$. This contradiction shows that indeed at most two edges of the form $[v, u]$ are clockwise bad at $v$. The same argument shows that if $u$ is a vertex of $G$ whose $x$-coordinate is maximum or minimum, then there is at most one edge incident with $u$ which is clockwise bad at $u$. Altogether, the total number of clockwise bad edges is bounded by $2+2 \cdot(n-2)=2 n-2$, completing the proof of Claim 1 .

Returning to our graph $G$ with $n$ edges and $6 n-5$ edges, which satisfies (2), define $G_{1}=G \partial, G_{2}=\partial G_{1}, G_{3}=G_{2} \partial$. Clearly, all the graphs $G_{1}, G_{2}$, and $G_{3}$ satisfy (2) and hence, by applying Claim 1 three times, we conclude that the number of edges of $G_{3}$ is at least $6 n-5-3(2 n-2)=1$. Let $e=\left[u_{1}, u_{2}\right]$ be an edge of $G_{3}$. Since $G_{3}=G_{2} \partial,\left[u_{1}, u_{2}\right]$ is a counterclockwise good edge of $G_{2}$. Consequently, there is an edge $\left[u_{1}, v_{1}\right]$ of $G_{2}$ such that the directed line $\vec{u}_{1} v_{1}$ is obtained from $\overrightarrow{u_{1} u_{2}}$ by rotating it counterclockwise around $u_{1}$ by an angle smaller than $\pi / 100$ (see Fig. 1). Similarly, there is an edge $\left[u_{2}, v_{2}\right]$ of $G_{2}$ with $\Varangle u_{1} u_{2} v_{2}<$ $\pi / 100$, as in Fig. 1 . Since $G_{2}=\partial G_{1}$ there are edges $\left[v_{1}, w_{1}\right]$ and $\left[v_{2}, w_{2}\right]$ of $G_{1}$ with $\Varangle u_{1} v_{1} w_{1}<\pi / 100$ and $\Varangle u_{2} v_{2} w_{2}<\pi / 100$, as in Fig. 1 . (It is worth noting that it may be, for example, that $\left[v_{1}, w_{1}\right]$ intersects both $\left[v_{2}, u_{2}\right]$ and $\left[v_{2}, w_{2}\right]$, or even that $w_{1}=v_{2}$.) Finally, as $G_{1}=G \partial$ there are edges $\left[w_{1}, x_{1}\right]$ and $\left[w_{2}, x_{2}\right]$ of $G$, with $\Varangle v_{1} w_{1} x_{1}<\pi / 100$ and $\Varangle v_{2} w_{2} x_{2}<\pi / 100$, as in Fig. 1. All seven edges $\left[x_{2}, w_{2}\right]$, $\left[w_{2}, v_{2}\right],\left[v_{2}, u_{2}\right],\left[u_{2}, u_{1}\right],\left[u_{1}, v_{1}\right],\left[v_{1}, w_{1}\right]$, and $\left[w_{1}, x_{1}\right]$, depicted in Fig. 1, belong to $G$. To complete the proof we show that they must contain three pairwise disjoint edges. Without loss of generality we may assume that $\Varangle u_{2} u_{1} v_{1} \geq \Varangle u_{1} u_{2} v_{2}$. 


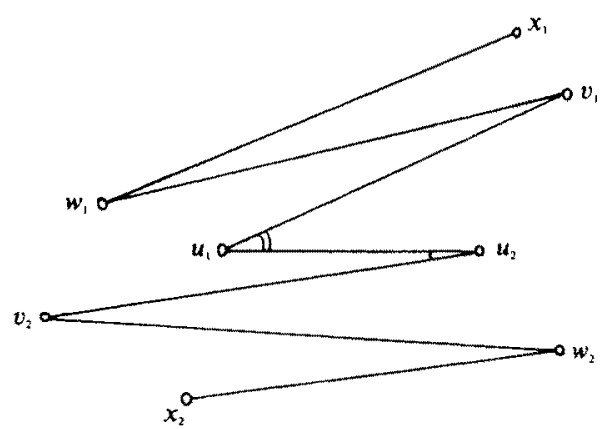

Fig. 1

If the length $l\left[v_{2}, u_{2}\right]$ of the segment $\left[v_{2}, u_{2}\right]$ satisfies $l\left[v_{2}, u_{2}\right] \geq l\left[u_{1}, u_{2}\right]$ (as is the case in Fig. 1), then we can easily check that $\left[x_{2}, w_{2}\right],\left[v_{2}, u_{2}\right]$, and $\left[u_{1}, v_{1}\right]$ are three pairwise disjoint edges. Otherwise, $l\left[v_{2}, u_{2}\right]<l\left[u_{1}, u_{2}\right]$ and then it is easy to check that $\left[v_{2}, w_{2}\right],\left[u_{1}, u_{2}\right]$, and $\left[w_{1}, v_{1}\right]$ are three pairwise disjoint edges. Therefore, in any case, $G$ contains three pairwise disjoint edges, completing the proof of Theorem 1.

\section{Acknowledgment}

We would like to thank Y. Kupitz, I. Krasikov, and M. A. Perles for helpful discussions.

\section{References}

[AA] J. Akiyama and N. Alon, Disjoint simplices and geometric hypergraphs, Proc. 3rd New York Conference on Combinatorial Mathematics, Annals of the New York Academy of Sciences, to appear.

[AH] S. Avital and H. Hanani, Graphs, Gilyonot Lematematika 3(2) (1966), 2-8 (in Hebrew).

[Er] P. Erdös, On sets of distances of $n$ points, Amer. Math. Monthly 53 (1946), 248-250.

[Ku] Y. S. Kupitz, Extremal Problems in Combinatorial Geometry, Aarhus University Lecture Notes Series, No. 53, Aarhus University, Denmark, 1979.

[Pe] M. A. Perles, Unpublished notes.

Received May 19, 1988. 